\title{
Assessment of Amplification Benefit for a Subject with Cochlear Dead Regions
}

\author{
E. SkrodzKA ${ }^{a}$ AND D. HoJAN-JEZIERSKA ${ }^{b *}$ \\ ${ }^{a}$ Institute of Acoustics, A. Mickiewicz Univ., Umultowska 85, 61-614 Poznań, Poland \\ ${ }^{b}$ Dept. of Biophysics, Poznań Univ. of Medical Sciences, Fredry 10, 61-701 Poznań, Poland
}

\begin{abstract}
The purpose of the study was to determine the optimal hearing aid fitting procedure for a patient with well diagnosed high-frequency dead regions in both cochleas. The patient reported non-symmetrical hearing problems of sensorineural origin. The presence and frequency limits of dead regions was measured using the Threshold Equalizing Noise test (TEN). For binaural amplification four pairs of hearing aids were used. Three pairs of hearing instruments used were independent pairs with similar hearing aids in each pair. One pair of hearing aids was a pair of dependent devices with an ear-to-ear function. Two fitting methods were used: DSLi/o and NAL-NL1 and four different strategies of fitting were tested: the initial fitting based on the DSLi/o or NAL-NL1 method with necessary loudness corrections, the second fitting taking into account all available functions of hearing instruments, the third fitting (based on the second one) but with significantly reduced amplification well above one octave of frequency inside dead region and the final fitting with significantly reduced gain slightly below one octave inside dead regions. The results of hearing aids fitting were assessed using the Abbreviated Profile of Hearing Aid Benefit procedure (APHAB).
\end{abstract}

PACS numbers: 43.66 Ts

\section{Introduction}

By definition a dead region is a region in the cochlea where inner hair cells (IHCs) and/or neurons are functioning so poorly that a tone producing peak vibration in that region is detected by off-place listening [1]. The majority of dead zones are high-frequency dead regions. A frequency limit of the dead region is defined in terms of the characteristic frequencies of the IHCs and/or neurons immediately adjacent to the this region (off-place listening). Therefore, it is not possible to determine from the pure tone audiogram alone whether or not a subject has a dead region. The newest data of Vinay and Moore [2] indicate that about $59 \%$ of ears had a dead region when the absolute threshold was above $70 \mathrm{~dB}$ HL in the frequency range $500-4000 \mathrm{~Hz}$. This result seems to be rather overestimated when a "clinically significant" dead region is taken into account. A clinically significant dead region is defined as a dead region that influences selection of amplification characteristics [3]. Thus, as an effective frequency range of many hearing instruments is limited to $4-5 \mathrm{kHz}$, the dead region is clinically significant if it extends down to at least $2 \mathrm{kHz}$. With such frequency limit the prevalence of dead regions is lowered to $25-30 \%$. Effects of dead regions on speech intelligibility were reported by Vickers et al. [4], Baer et al. [5] and Hojan-Jezierska et al. [6]. For adults with high-frequency dead

* corresponding author; e-mail: agamon63@wp.pl regions, there is little or no benefit to speech discrimination from amplifying frequencies well inside a dead region but there may be some benefit (not only in intelligibility of speech) amplifying frequencies up to 50-100\% above the estimated edge frequency of the dead region. For patients without high-frequency dead regions, amplification of the high frequencies usually is beneficial.

Our subject had dead regions in both ears. Frequency limits of them were measured using the standard the Threshold Equalizing Noise (TEN) test $[3,7]$.

The aims of the presented study were:

— to check the hearing benefit resulting from different amplification methods and strategies,

- to check which type of modern hearing instruments is more beneficial for such case of dead region: high-technology based hearing instruments with many innovative features or less-technology advanced instruments with limited number of additional functions.

\section{Subject, hearing instruments and the method}

Our subject was a woman, 40-years old, with dead regions in both cochleas, employed. The tonal audiogram of the subject as well as the results of the TEN test are shown in Fig. 1.

The hearing loss shown in Fig. 1 is severe. It is a result of medical treatment by ototoxic medicines in early 


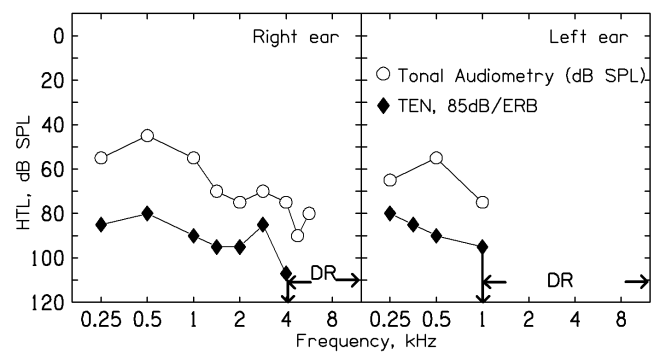

Fig. 1. Tonal audiogram of the subject, left ear.

childhood. A method for dead region diagnosis was the Threshold Equalizing Noise (TEN) method [3]. In the right ear the dead region began at about $4 \mathrm{kHz}$ and in the left ear — at about $1 \mathrm{kHz}$.

We used four pairs of behind the ear (BTE) digital hearing instruments, denoted $\mathrm{H} 1, \mathrm{H} 2, \mathrm{H} 3$ and $\mathrm{H} 4^{\dagger}$, described in Table. The subject was not informed about a price and specific technical parameters of the fitted instrument, listed in Table.

Two fitting methods were used: DSLi/o [8] and NALNL1 [9]. The DSLi/o method is based on the tonal audiogram and does not take into account the presence of a dead region. The NAL-NL1 method is also based on the tonal audiogram and does not comprise the presence of the dead region, too. However, it takes into account the general, well known fact, that people with high-frequency hearing loss get progressively less benefit from amplification of the high frequencies as the hearing loss increases. It is worthwhile to add that at the moment there is no a popular and faithful fitting formula incorporating the fact of a dead zone existence. For each fitting method four fitting strategies were tested. In the first (initial) of them the fitting method was DSLi/o or NAL-NL1 and only adjustment of loudness was allowed. In the second strategy the hearing instrument gain was adjusted using all it's available features to obtain subjectively assessed maximum of speech intelligibility and comfort. In the third fitting (based on the second one) amplification was significantly reduced well above one octave of frequency inside dead region. In the final fitting gain was significantly reduced slightly below one octave inside dead regions. Every pair of hearing instruments with a given fitting strategy and fitting method was tested during two weeks.

Subjective hearing aid performance was measured with the Abbreviated Profile of Hearing Aid Benefit (APHAB) $[3,10]$ questionnaire. The APHAB is a 24-item questionnaire consisting of four subscales: Ease of Communication (EC), Reverberation (RV), Background Noise (BN), and Aversioness (AV). These subscales assess the benefit

\footnotetext{
${ }^{\dagger} \mathrm{H} 1$ - Siemens Artis SP, H2 - Siemens Artis SP e2e, H3 -
} AudioService Riva 2HP, H4 - AudioService Monza2 DuoT
TABLE

Features and additional systems in hearing aids used in the experiment.

\begin{tabular}{c|l}
\hline $\begin{array}{c}\text { Hearing } \\
\text { aid }\end{array}$ & \multicolumn{1}{|c}{ Features and additional systems } \\
\hline H1 & $\begin{array}{l}\text { BTE, digital, programmable, 6 frequency chan- } \\
\text { nels, acoustic feedback reduction system, adaptive } \\
\text { noise reduction system in 6 channels, 3 compres- } \\
\text { sion channels }\end{array}$ \\
\hline H2 & $\begin{array}{l}\text { BTE, the same as H1 but with ear-to-ear func- } \\
\text { tion, which synchronizes the signal processing of } \\
\text { both hearing instruments so that they operate si- } \\
\text { multaneously as one unit. }\end{array}$ \\
\hline H3 & $\begin{array}{l}\text { BTE, digital, programmable, 4 frequency chan- } \\
\text { nels, adaptive noise reduction system, acous- } \\
\text { tic feedback reduction system, directional micro- } \\
\text { phone }\end{array}$ \\
\hline H4 & $\begin{array}{l}\text { BTE, digital, programmable, 7 frequency chan- } \\
\text { nels, acoustic feedback reduction system, adaptive } \\
\text { noise reduction system, } 4 \text { compression channels, } \\
\text { directional microphone }\end{array}$
\end{tabular}

derived from hearing aid use with respect to communication under ideal listening conditions (low noise/reverberation with visual cues available), communication under quiet, but poor, listening conditions (e.g., reverberation, soft speech, and visual cues unavailable), communication in noisy situations, and negative reactions to environmental sounds. The APHAB test was fulfilled after every 2-week period of experiment. Each time questions were ask for situations with and without amplification.

\section{Results and discussion}

In Fig. 2 examples of predicted insertion gains for the left ear, NAL-NL1 fitting procedure, four tested fitting strategies and for input sound pressure level equal to $65 \mathrm{~dB}$ (average level of speech) are shown for the hearing instrument H2. Similar course of gain curves was obtained for the DSLi/o method and for the right ear (not presented in the paper).

As can be noticed the initial NAL-NL1 fitting method was not optimal, and further adjustment of instruments was always necessary. After initial fitting insertion gain was always underestimated in low frequencies and overestimated in the high frequency range. For instruments H2 and NAL-NL1 method the initial fitting was completely different from gains predicted by other strategies. For strategies 2-4 gain curves did not differ too much from each other, they were significantly limited in the frequency domain and they demanded very serious changes in amplification parameters when compared to initial settings. It is clear from Fig. 2 and all collected results that final fitting was always necessary. Similar situation was observed for all tested pairs of hearing aids and both fitting methods. Differences in the insertion gain for final 


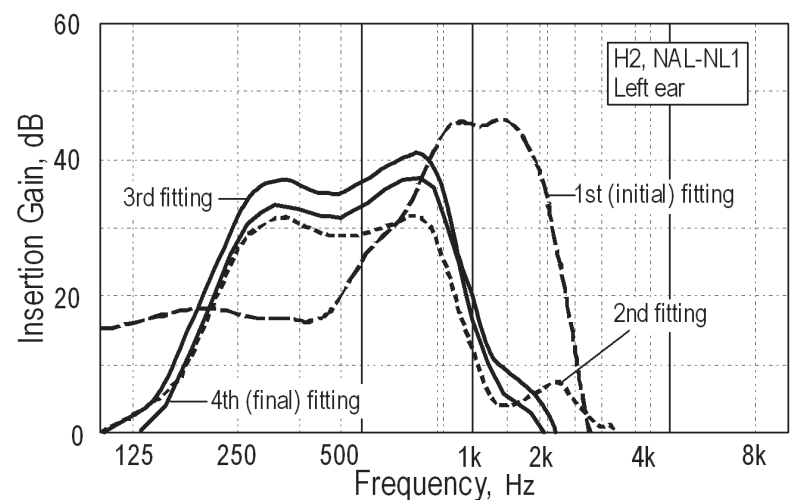

Fig. 2. Examples of predicted insertion gains for four strategies used, the hearing aid H2, NAL-NL1 fitting method and the left ear.

fitting for all tested pairs of hearing aids are presented in Fig. 3.

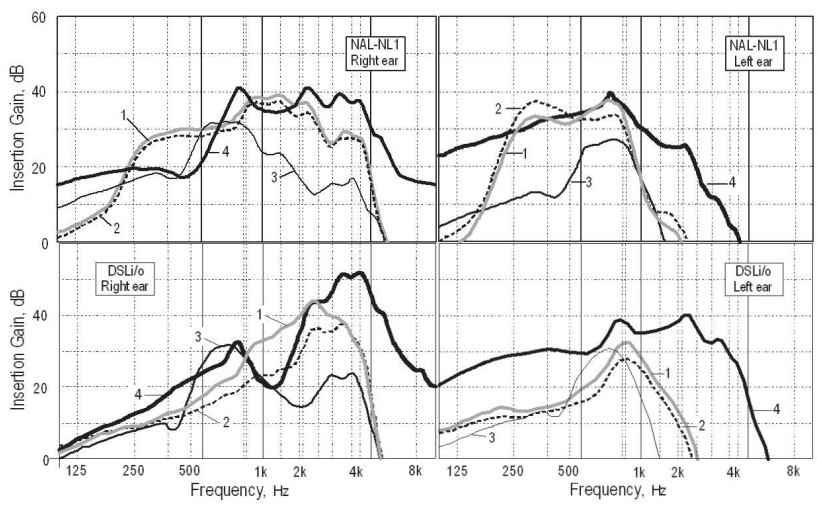

Fig. 3. Examples of final fitted insertion gains for NAL - NL1 and DSLi/o method and for both ears. Numbers denote hearing aids listed in Table. More details can be found in the text.

In the upper row results for NAL-NL1 method are shown. In the bottom row results for DSLi/o method are presented. Left column contains results for the right ear, the right column - results for the left ear. Numbers 1-4 denote insertion gains for hearing aids 1-4 listed in Table. As can be noticed from Fig. 3 final insertion gains predicted by the NAL-NL1 method are significantly lower then those predicted by the DSLi/o method, especially in high frequency range. Although curves in Fig. 3 are different, it is not possible from them to asses hearing problems and amplification benefit. Therefore, for each tested situation the APHAB test was performed.

In Figs. 4-5 results of the APHAB test are shown. The APHAB questionnaire was administered in computer-and-mouse format. In cases where an item was not applicable, the subject was instructed to make an estimation of how well she thought she would understand speech or how aversive sounds might be in that situation. Benefit was calculated by comparing the subject's reported difficulty in the unaided condition with their amount of difficulty when using amplification. In the APHAB test binaural hearing was assessed.

As can been seen from Figs. 4-6 that the smallest number of problems and the greatest benefit was generally reported for the final fitting for DSLi/o fitting method and pairs of hearing aids $\mathrm{H} 1-\mathrm{H} 3$.

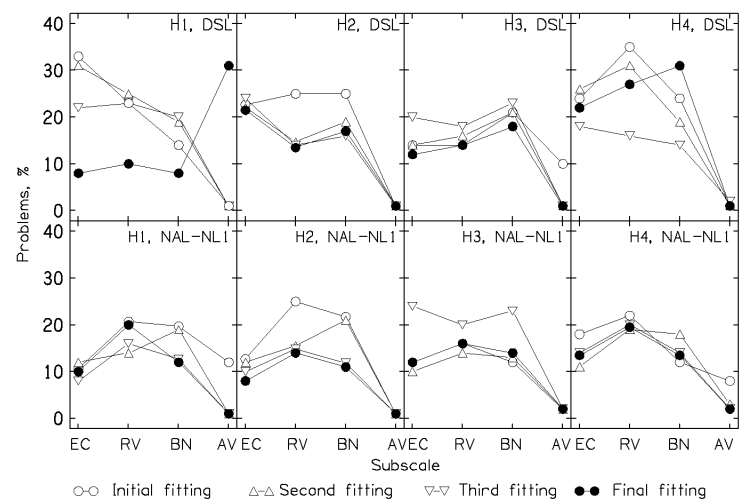

Fig. 4. Results of the APHAB test - subjectively reported problems in hearing aid performance.

In the case of the NAL-NL1 method situation was more complicated. In majority of APHAB's categories hearing problems after final fitting were assessed as to be either lower or similar to problems reported after previous stages of fitting. Benefit was assessed as lower than in the case of DSLi/o. However, even in these cases, the final fitting based on amplification reduction slightly below one octave inside dead regions seems to be desirable. In three main $\mathrm{APHAB}$ categories (EC, $\mathrm{RV}$ and $\mathrm{BN}$ ) the overall lowest number of problems and the best benefit score was reported for the pair of instruments H1, fitted using DSLi/o method and for final fitting. Relatively high percentage of problems reported after final fitting in AV category was probably cause by the fact that the pair H1 with DSLi/o method was fitted as the first and the subject heard environmental noise, which had not been heard before. The pair of hearing instruments denoted as $\mathrm{H} 1$ in Table is not as technically advanced as other tested pairs (i.e. H2 and H3). It is too far to come to the conclusion, that hearing instruments with many frequency channels and equipped with sophisticated systems are not suitable for subjects with dead regions. But form our investigation it is clear that the less technically sophisticated hearing aids may be a cheaper alternative for "dead region" patients.

Comparison of the APHAB test results for the final fitting and for all tested situations is shown in Fig. 6 . From this figure it is again clearly visible, the our subject assessed the pair H1 (without eat-to-ear function) fitted using DSLi/o method as the best from the tested set. We would like to stress, that in the particular case of our subject the pair $\mathrm{H} 1$ with DSLi/o method was the best choice and that it might not be a rule for other subjects with dead regions. What is more, the benefit 


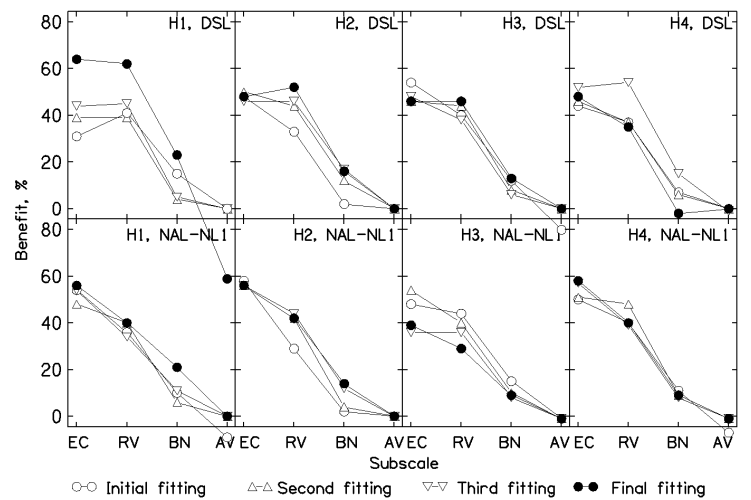

Fig. 5. Results of the APHAB test - benefit from amplification.

of $68 \%$ does not mean, that the speech intelligibility is also $68 \%$. It only means, that for this benefit value, the speech perception is the best possible.

The presented work is a continuation of our previous paper, where the same subject was tested, but only two pairs of hearing instruments were tested [11].

Only one subject was tested in our investigation. This number is obviously not appropriate for any statistical considerations. However, similar situations, when only a few ears were investigated trying to formulate general conclusions, can be found in some reports $[3,12]$.
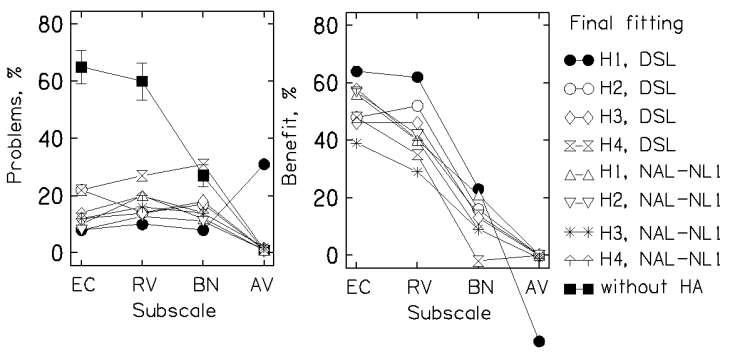

Fig. 6. Results of the APHAB test after final fitting.

\section{Conclusions}

From analysis of the case and the experiment performed on the subject with well defined limits of cochlear dead regions in both ears we conclude, that:

- the DSLi/o fitting method of hearing aid was optimal for this case; the alternative methods would be NAL-NL1 or NSLE [6],

- limitation of the frequency range of amplification to no more than one octave above a low frequency limit of a dead region was always desirable and was beneficial to speech intelligibility and some aspects of acoustic comfort and life quality,

- the pair of independent hearing aids denoted as H1 (without ear-to-ear function) with DSLi/o fitting method was the most beneficial. The pair of instruments with ear-to-ear function and DSLi/o or NAL-NL1 fitting method denoted as H2, as well as other two pairs of independent hearing instruments denoted as H3 and H4 were less beneficial.

- It is possible that the modern, technically advanced hearing instrument with many frequency channels will be helpful, too. However, the possibility of application of less sophisticated and cheaper ones has not been rejected.

\section{Acknowledgments}

The work was partially supported by The National Center of Research and Development grant No. N R11 000804 .

We are grateful to Audio SAT Sp. z o.o., Poznań, Poland for granting hearing aids used in the experiment and technical help.

\section{References}

[1] B.C.J. Moore, Ear and Hearing 25, 98 (2004).

[2] S.N. Vinay, B.C.J. Moore, Ear and Hearing 28, 231 (2007).

[3] B.C.J. Moore, M. Huss, D.A. Vickers, B.R. Glasberg, J.I. Alcantara, British Journal of Audiology 34, 205 (2000).

[4] D.A. Vickers, B.C.J. Moore, T. Baer, J. Acoust. Soc. Amer. 110, 1164 (2001).

[5] T. Baer, B.C.J. Moore, K. Kluk, J. Acoust. Soc. Amer. 112, 1133 (2002).

[6] D. Hojan-Jezierska, E. Skrodzka, E. Hojan, Arch. Acoust. 32(3), 441 (2007).

[7] K.J. Munro, Phonak Focus 38, (2007).

[8] L.E. Cornelisse, R.C. Seewald, D.G. Jamieson, J. Acoust. Soc. Amer. 97, 1854 (1995).

[9] H. Dillon, Hearing Journal 52, 10 (1999).

[10] R. Cox, G. Alexander, Ear and Hearing 16, 176 (1995).

[11] D. Hojan-Jezierska, E. Skrodzka, Polish Journal of Medical Physics and Engineering (2009) (in press).

[12] A. Karczewska-Nabelek, Arch. Acoust. 32, 505 (2007). 